\title{
HAUSDORFF MEANS WITH CONVEX MASS FUNCTIONS
}

H. S. WALL

ThEOREM A. If $[H, \phi]$ is a regular Hausdorff mean and $(C, 1)$ the Cesaro mean of order 1 , then the following two statements are equivalent:

(i) $[H, \phi]$ includes $(C, 1)$,

(ii) $\phi=\phi_{1}-\phi_{2}+i \phi_{3}-i \phi_{4}$, where each of $\phi_{1}, \phi_{2}, \phi_{3}$, and $\phi_{4}$ is a nonnegative, nondecreasing and convex function on the interval $[0,1]$.

1. Proof that (i) implies (ii). In order that the regular Hausdorff mean $[H, \phi]$ should include $(C, 1)$, it is necessary and sufficient that there should exist a regular mass function $f$, such that

$$
\phi(x)=f(x)+x \int_{x}^{1} \frac{1}{t} d f(t), \quad \text { for } x>0 .
$$

This is a particular case of Theorem 3.1, p. 196, of [2].1

Suppose that $[H, \phi]$ is a regular Hausdorff mean which includes $(C, 1)$, and that $f$ is a regular mass function satisfying (1.1). Then, $f=f_{1}-f_{2}+i f_{3}-i f_{4}$, where each of $f_{1}, f_{2}, f_{3}$, and $f_{4}$ is a nondecreasing function in $[0,1]$. If

$$
K(x, t)=\left\{\begin{array}{lr}
x / t & \text { for } 0 \leqq x \leqq t, t>0, \\
1 & \text { for } x \geqq t \geqq 0,
\end{array}\right.
$$

then $K(x, t)$ is a nonnegative, nondecreasing, and convex function of $x$ for each nonnegative number $t$, and the equation (1.1) may be written: $\phi(x)=\int_{0}^{1} K(x, t) d f(t)$, for $x>0$. Suppose $\phi_{j}(x)=\int_{0}^{1} K(x, t) d f_{j}(t)$, for $j=1,2,3,4$, so that $\phi=\phi_{1}-\phi_{2}+i \phi_{3}-i \phi_{4}$, and $\phi_{j}$ is nonnegative and nondecreasing. If $u, v, w$, is an increasing sequence of numbers in $[0,1]$, then

$$
\left|\begin{array}{lll}
u, & \phi_{j}(u), & 1 \\
v, & \phi_{j}(v), & 1 \\
w, & \phi_{j}(w), & 1
\end{array}\right|=\int_{0}^{1}\left|\begin{array}{lll}
u, & K(u, t), & 1 \\
v, & K(v, t), & 1 \\
w, & K(w, t), & 1
\end{array}\right| d f_{j}(t) \leqq 0,
$$

so that $\phi_{j}$ is convex. Hence, (i) implies (ii).

2. Proof that (ii) implies (i). In order to prove that (ii) implies (i), we make use of some elementary properties of convex functions. Suppose first that $\phi$ is a regular mass function which is nonnegative, non-

Presented to the Society, December 28, 1952; received by the editors November 14, 1952 and, in revised form, January 6, 1953.

${ }^{1}$ We use the notation and terminology of [2]. 
decreasing, and convex on $[0,1]$. If $0<x<1$, then $\phi(x)$ has a righthand derivative $\phi_{r}^{\prime}(x)$ and a left-hand derivative $\phi_{i}^{\prime}(x)$. If $\phi^{\prime}(x)$ denotes $\left(\phi_{r}^{\prime}(x)+\phi_{i}^{\prime}(x)\right) / 2$, then $\phi^{\prime}$ is a nonincreasing function, and if $t$ is a number between 0 and 1 , then no point of the graph of $\phi$ is above the straight line whose equation is $y=\phi(t)+(x-t) \phi^{\prime}(t)$. It follows that the $y$-intercept, $\phi(t)-t \phi^{\prime}(t)$, of this straight line, is nonnegative and is a nondecreasing function of $t$. Inasmuch as $0 \leqq t \phi^{\prime}(t) \leqq \phi(t)$ for $0<t<1$, and $\phi(0)=\phi(0+)$, then $t \phi^{\prime}(t) \rightarrow 0$ as $t \rightarrow 0+$.

Suppose

$$
f(t)=\left\{\begin{array}{lr}
\phi(t)-t \phi^{\prime}(t) & \text { for } 0<t<1, \\
0 & \text { for } t=0, \\
1 & \text { for } t=1 .
\end{array}\right.
$$

Then, $f$ is a regular mass function which is nondecreasing on $[0,1]$. The function $t \phi^{\prime}(t)$, which we define to be 0 for $t=0$ and for $t=1$, is the difference of two nondecreasing functions on $[0,1]$.

Now, if $0<x \leqq 1$, then

$$
\begin{aligned}
\int_{0}^{1} K(x, t) d f(t) & =f(x)+x \int_{x}^{1} \frac{1}{t} d \phi(t)-x \int_{x}^{1} \frac{1}{t} d\left[t \phi^{\prime}(t)\right] \\
& =f(x)+x \int_{x}^{1} \frac{1}{t} d \phi(t)+x \phi^{\prime}(x)-x \int_{x}^{1} \frac{\phi^{\prime}(t)}{t} d t \\
& =\phi(x) .{ }^{2}
\end{aligned}
$$

Hence, by the first statement in $\$ 1,[H, \phi]$ includes $(C, 1)$.

In the general case, suppose $\phi=\phi_{1}-\phi_{2}+i \phi_{3}-i \phi_{4}$, where each of $\phi_{1}, \phi_{2}, \phi_{3}$, and $\phi_{1}$ is a nonnegative, nondecreasing, and convex function on $[0,1]$. We may suppose that $\phi_{j}(0)=\phi_{j}(0+)=0$. If

$$
f_{j}(t)=\left\{\begin{array}{lr}
\phi_{j}(t)-t \phi_{j}{ }^{\prime}(t) & \text { for } 0<t<1, \\
0 & \text { for } t=0, \\
\phi_{j}(1) & \text { for } t=1,
\end{array}\right.
$$

then the function $f_{1}-f_{2}+i f_{3}-i f_{4}$, denoted by $f$, is a regular mass function, and we find by the preceding that $\phi(x)=\int_{0}^{1} K(x, t) d f(t)$ for $x>0$, so that $[H, \phi]$ includes $(C, 1)$.

This completes the proof of Theorem A.

REMARK 2.1. About the same argument used in the proof of Theorem $A$ shows that in order for a function $\phi(x)$ to be nonnegative, convex, and bounded for $x>0$, it is necessary and sufficient that there

${ }^{2}$ Cf. the argument in [1] to prove $\int_{x}^{1}(1 / t) d \phi(t)=\int_{x}^{1}\left(\phi^{\prime}(t) / t\right) d t$. 
should exist a function $f(t)$ which is nondecreasing and bounded for $t \geqq 0$, such that $\phi(x)=\int_{0}^{\infty} K(x, t) d f(t)$ for $x>0$. In my original proof of Theorem A, I first deduced this theorem, by a rather lengthy argument, from an integral representation which Blaschke and Pick [1] found for a function which is nonnegative and convex in a (finite) segment $(a, b)$. The referee suggested an easier proof [that (ii) implies (i)], based upon a different formulation ((i), p. 194, of [2]) of the Hausdorff inclusion problem.

REMARK 2.2. Necessary and sufficient conditions were given in [2] in order that a regular Hausdorff mean $[H, \phi]$ should include the Cesaro mean $(C, n)$ of positive integral order $n$. It was stated there that some parts of the conditions may be redundant. For the case $n=1$, we see that these conditions may be reduced to the following: (1) $\phi_{r}^{\prime}(x)$ exists for $0<x<1$ and $\phi_{l}^{\prime}(x)$ exists for $0<x \leqq 1,(2) x \phi^{\prime}(x)$ $\rightarrow 0$ as $x \rightarrow 0$, and (3) $x \phi^{\prime}(x)$ is of bounded variation on $[0,1]$. Denote $\int_{0}^{1} K(x, t) d f(t)$ by $L(f)$. In order for the regular Hausdorff mean $[H, \phi]$ to include $(C, n)$, it is necessary and sufficient that there should exist a regular mass function $f$ such that $\phi=L^{n}(f)$. Suppose $M_{1}$ is the set of all regular mass functions $\phi$ such that $[H, \phi]$ includes $(C, 1)$, and, for each positive integer $n, M_{n+1}$ is the set of all functions $\phi$ such that $\phi$ and $\phi-x \phi^{\prime}$ belong to $M_{n}$. In order for the regular Hausdorff mean $[H, \phi]$ to include $(C, n)$, it is necessary and sufficient that $\phi$ should belong to $M_{n}$. A function sufficiently "flat" at 1, e.g., 1 $-\exp (x /(x-1))$, belongs to every one of the sets $M_{n}{ }^{3}$

REMARK 2.3. If $\phi$ is a regular mass function, $\phi^{\prime}(1)$ exists, and $\phi$ is convex or concave on $[0,1]$, then $\phi \in M_{1}$. Professor G. G. Lorentz has told me that his student, J. P. Leah (Ph.D. thesis, Toronto, 1952) connected convexity or concavity of the mass functions with the Hausdorff means which include $(C, 1)$ "totally" (i.e., if $(C, 1)$ assigns to a real sequence a value which is finite or $+\infty$ or $-\infty$, then $[H, \phi]$ assigns the same value).

\section{BIBLIOGRAPHY}

1. W. Blaschke and G. Pick, Distanzschätzungen in Funktionenraum II, Math. Ann. vol. 77 (1915) pp. 277-302.

2. H. L. Garabedian, Einar Hille, and H. S. Wall, Formulations of the Hausdorff inclusion problem, Duke Math. J. vol. 8 (1941) pp. 193-213.

\section{Department of Pure Mathematics, University of Texas}

${ }^{3}$ This example was given by H. L. Garabedian (Bull. Amer. Math. Soc. vol. 48 (1942) pp. 124-127). F. Hausdorff (Math. Zeit. vol. 9 (1921) pp. 74-109, 280-299) had (less simple) examples of such functions. The set of all such functions may be characterized as the set $M$ of all regular mass functions such that if $\phi \in M$ then $\phi-x \phi^{\prime} \in M$. 